\title{
Augmented Bicarbonate Reabsorption by Both the Proximal and Distal Nephron Maintains Chloride-deplete Metabolic Alkalosis in Rats
}

\author{
Donald E. Wesson \\ With the technical assistance of Herbert Babino \\ Veterans Administration Medical Center, Baylor College of Medicine, Houston, Texas 77211
}

\begin{abstract}
Whether augmented bicarbonate reabsorption by renal tubular epithelium contributes to the maintenance of chloride-deplete metabolic alkalosis is not clear. This study used free-flow micropuncture to investigate bicarbonate reabsorption by surface nephron segments in a rat model of diuretic-induced alkalosis compared to control. The proximal and distal nephron of the alkalotic animals had higher values for both delivered load to and absolute reabsorption from these segments. The proximal tubules of alkalotic and control animals had similar values for the slopes of the linear regression of delivered load vs. reabsorption and for the bicarbonate tubular fluid to plasma (TF/P) ratio at the late proximal tubule. By contrast, the corresponding analysis for the distal segment of alkalotic animals revealed a greater slope $(0.98$ vs. $0.81, P<0.003)$ and a smaller bicarbonate TF/P ratio at the late distal tubule $(0.10$ vs. 0.16, $P<0.006$ ). The data indicate that augmented bicarbonate reabsorption by both the proximal and distal nephron contributes to maintaining the alkalosis of this model. The data suggest primary stimulation of bicarbonate reabsorption in the distal nephron and load-dependent reabsorption in the proximal tubule.
\end{abstract}

\section{Introduction}

The mechanism for the maintenance of chloride-deplete metabolic alkalosis remains unresolved. Whether augumented bicarbonate reabsorption by renal tubular epithelia contributes to this acid-base disorder has yet to be firmly established. Technical considerations have hindered interpretation of previous micropuncture studies, which have investigated renal tubular bicarbonate reabsorption in various animal models of this disorder. Firstly, proximal tubule bicarbonate reabsorption has most often been assessed using quantitative proximal tubule fluid collections that use an oil block (1-6). This proximal tubule collection technique maximally stimulates the tubuloglomerular feedback (TGF) ${ }^{1}$ response $(7,8)$ and thereby potentially introduces artifact to the determination of tubular bicarbonate reabsorption. Secondly, most studies report reabsorptive data for only the proximal tubule and not for the loop

Address reprint requests to Dr. Donald E. Wesson, Assistant Chief, Renal Section (151-B), Veterans Administration Medical Center, 2002 Holcombe Boulevard, Houston, TX 77030.

Received for publication 27 September 1988 and in revised form 7 July 1989.

1. Abbreviations used in this paper: ECF, extracellular fluid; SNGFR, single nephron glomerular filtration rate; TF/P, tubular fluid/plasma ratio; TGF, tubuloglomerular feedback.

The Journal of Clinical Investigation, Inc

Volume 84, November 1989, 1460-1469 segment nor for the surface distal nephron (4-6). Thirdly, investigators have studied tubular bicarbonate reabsorption using various models of chloride-deplete alkalosis. These models have been infrequently characterized, hindering comparison among various experimental models $(1,2,5,6)$. Finally, these models have been studied at various times after induction of the alkalosis, which also makes comparisons among studies difficult.

This free-flow micropuncture study investigated renal tubular bicarbonate reabsorption in Munich-Wistar rats with and without chloride-deplete metabolic alkalosis while addressing the technical problems discussed above. A model of diuretic-induced alkalosis has been studied at two different times after induction of the alkalosis and characterized as to whole animal electrolyte balance, inulin space, and GFR. Finally, bicarbonate reabsorption of nephron segments has been determined using a method that reduces stimulation of the TGF response and thereby yields a value that more closely resembles that occurring in situ. The data indicate that increased bicarbonate reabsorption by both the proximal and the distal nephron contributes to the maintenance of chloride-deplete metabolic alkalosis. Furthermore, the data suggest that the increased reabsorption by the distal nephron is due to a primary stimulation of bicarbonate reabsorption, whereas that in the proximal tubule is in response to an increase in filtered load of bicarbonate.

\section{Methods}

\section{Experimental procedures}

Male and female Munich-Wistar rats (Harlan Sprague Dawley, Houston, TX) weighing $210-280 \mathrm{~g}$ were used in all micropuncture experiments.

Below are outlined the metabolic alkalosis induction protocol, various preliminary studies, and the micropuncture protocol. Animal growth studies were done comparing experimental animals with untreated animals on standard rat chow as a gross estimation of the nutritional status of the experimental animals. Because disturbances of electrolyte balance may influence renal tubular bicarbonate reabsorption (9), electrolyte balance studies and muscle potassium measurements were done to characterize the environment in which the measured tubular bicarbonate reabsorption occurred and to allow for comparison with other models of chloride-deplete alkalosis. Inulin space determinations were done to qualitatively compare the extracellular fluid volumes of animals with and without alkalosis since disturbances of extracellular fluid (ECF) ${ }^{1}$ volume may influence renal bicarbonate reabsorption (10).

Induction of chloride-deplete metabolic alkalosis. Chloride-deplete metabolic alkalosis was induced in experimental animals using a method similar to that used by others $(5,6)$. Animals fed standard rat chow (Ralston Purina Co., St. Louis, MO) received three intraperitoneal injections of furosemide ( $10 \mathrm{mg} / \mathrm{kg}$ body wt/dose) at 12 -hour intervals and started on a low electrolyte diet (ICN Nutritional Biochemicals, Cleveland, $\mathrm{OH}$ ) and given distilled $\mathrm{H}_{2} \mathrm{O} .48 \mathrm{~h}$ after the first furosemide injection, the drinking water of control animals was 
changed to $40 \mathrm{mM} \mathrm{NaCl}$ plus $40 \mathrm{mM} \mathrm{KCl}$ and that of the alkalotic animals to $40 \mathrm{mM} \mathrm{NaHCO}_{3}$ plus $40 \mathrm{mM} \mathrm{KHCO}_{3}$. The low electrolyte diet was continued for the duration of the protocol.

Preliminary studies from this laboratory as well as published studies by other investigators (6) demonstrated that both whole kidney and single nephron GFR increased progressively after initiation of this protocol in the alkalotic animals and stabilized at $\sim 3 \mathrm{wk}$. Because GFR potentially plays a major role in maintaining metabolic alkalosis (4), some animals were studied early (1 wk) and others late (4 wk) into the protocol.

Animal growth studies. 27 rats of similar body weights were selected to determine the effect of the above induction protocol on animal growth. 10 control and 10 alkalotic animals underwent the above described protocol and the remaining 7 animals were kept on standard rat chow and distilled $\mathrm{H}_{2} \mathrm{O}$ and were not given furosemide. Daily body weights were measured for $28 \mathrm{~d}$, after which the animals were killed and left kidney weights were determined. The week times described represent time after initiation of the protocol, such that 1 wk represents $5 \mathrm{~d}$ after beginning the respective drinking solutions.

Electrolyte balance studies. Nine each of control and alkalotic animals underwent the induction protocol and were kept in metabolic cages for determination of net balances for sodium, potassium, and chloride. Electrolyte balance was the cumulative difference between intake and urinary excretion up to the time of study. Fecal electrolyte determinations were not done since previous investigators have shown fecal electrolyte excretion to be quite small (11). $1 \mathrm{wk}$ into the protocol, five control and five alkalotic animals underwent micropuncture (described below). The remaining four animals of each group completed 4 wk of the protocol.

Muscle potassium measurements. Muscle potassium concentration per fat-free dry solid weight of tissue was determined using the protocol described by other investigators (4). Thigh muscle was minced and then dried for $48 \mathrm{~h}$ to constant weight. The tissue was then extracted with ether, dried again, and reweighed. Nitric acid $(0.46 \mathrm{~N})$ digestion was done for $48 \mathrm{~h}$ with constant mild agitation. The supernatant potassium concentration was determined using flame photometry.

Micropuncture protocol. Overnight food-fasted rats were prepared for micropuncture as described in previous studies from this laboratory $(8,12)$. All animals received simulated rat plasma (Ringer's bicarbonate plus $40 \mathrm{~g} /$ liter of BSA) as a $1.0 \%$ body weight bolus followed by a maintenance infusion of $0.5 \%$ body weight per hour. This protocol maintained arterial hematocrit and protein concentration at baseline levels.

Micropuncture samples were obtained from the late proximal tubule of random nephrons, and from the late distal, then early distal, tubule of the same nephron in paired fashion. The tubule segments were localized after bolus injection of Food Dye and Coloring (FD \& C) green dye as previously described $(8,12)$. A localized early distal tubule was indicated by placing an FD \& $\mathrm{C}$ green dye-filled perfusion pipette over it of o.d. 2-3 $\mu \mathrm{m}$ mounted on a micromanipulator. A late distal tubule was localized in the vicinity of the designated early distal tubule and was similarly indicated with a collection pipette of o.d. 6-8 $\mu \mathrm{m}$ filled with Sudan black-stained, water-equilibrated, and $6.7 \%$ $\mathrm{CO}_{2}$-bubbled mineral oil. The perfusion pipette positioned over the previously indicated early distal tubule was then inserted into the tubule and a small amount of FD \& C green dye was injected into the tubule to insure continuity between it and the subsequently localized late distal tubule. If these early and late distal segments were not continuous, other adjacent late distal tubules were localized that were continuous with the localized early distal tubule. Then, a small volume of Sudan black-stained mineral oil of 3-4 tubular diameters in length was injected into the identified late distal tubule and aspiration of the tubular fluid was begun, the collection of which lasted 10-15 min. After the late distal collection was complete, an oil-filled collection pipette of similar size was inserted into the identified early distal tubule and a sample obtained in similar fashion for 5-6 min. Late proximal collections were done using two methods: $(a)$ using an oilfilled pipette of o.d. 8-10 $\mu \mathrm{M}$, an oil block of 3-4 tubular diameters in length was injected and tubular fluid was aspirated as described for distal collections for 2-3 min. This is the proximal collection method used by most investigators. (b) In addition, separate proximal tubules were punctured without an oil block using oil-filled pipettes of o.d. 3-4 $\mu \mathrm{M}$. The purpose of these two proximal tubule collection methods was to collect with maximum and minimum interruption of fluid flow to the loop of Henle for maximum and minimum stimulation of the TGF response. Stimulation of the TGF response causes an increase in single nephron (SN) GFR $(7,8)$ and an increase in SNGFR may not be associated with maintenance of glomerular tubular balance for bicarbonate reabsorption (13). The two techniques for fluid collection from the proximal tubule thus were used in an effort to detect an effect of collection technique on bicarbonate reabsorption calculated for the proximal tubule. The "no-block" collections were timed and were obtained using capillary action or very gentle aspiration and were collected at a rate of 4-5 $\mathrm{nl} / \mathrm{min}$ for 5-6 min. As was done with other tubular collections, collection rate for these "no-block" collections was determined by dividing the collected volume by the time of collection. The rate of fluid removal from the late proximal tubule using the "no-block" technique allowed for 50-70\% of the fluid flow calculated to occur at this site in situ (see Calculations and Table III) to flow into the loop of Henle of all groups. The residual fluid flow (in situ late proximal flow rate minus the $4-5 \mathrm{nl} / \mathrm{min}$ fluid collection) was at least 5 $\mathrm{nl} / \mathrm{min}$ for all groups studied. In vivo microperfusion studies show that this rate of fluid flow into the loop of Henle leads to less stimulation of the TGF response compared with zero flow (7). The "no-block" technique therefore allows for collection of proximal tubule fluid with less stimulation of the TGF response than does the "blocked" technique. Thus, any potential artifactual influences of the TGF response on bicarbonate reabsorption by the proximal tubule would be expected to be less with the "no-block" compared with the "blocked" technique. Bicarbonate reabsorption by the proximal tubule was calculated (see below) using each method of fluid collection for comparison. Both proximal and distal tubule collections were followed by injection of latex casts and subsequent $6 \mathrm{~N} \mathrm{HCl}$ digestion of the excised kidney for $60 \mathrm{~min}$ at $37^{\circ} \mathrm{C}$. The length of the dissected cast was measured under a microscope with an eye micrometer for determination of surface tubule length.

Inulin space determination. Inulin space determinations were done as described by other investigators (14) as comparative estimates of extracellular fluid volume in 20 control and 20 alkalotic animals. 10 animals of each group were done $1 \mathrm{wk}$ into the induction protocol and the remainder were done after $4 \mathrm{wk}$. The animals were surgically prepared as described above except that no flank incision was made. Volume replacement was done using simulated rat plasma as described above to maintain arterial hematocrit and plasma protein concentration unchanged from baseline. $100 \mu \mathrm{Ci}$ of exhaustively dialyzed $\left[{ }^{3} \mathrm{H}\right]$ inulin dissolved in $1 \mathrm{ml}$ of $5 \%$ dextrose in water (D5W) was injected intravenously and followed by a $0.5-\mathrm{ml}$ D5W flush. Tail vein blood for $\left[{ }^{3} \mathrm{H}\right]$ inulin determinations were obtained in capillary tubes every 10 min for $60 \mathrm{~min}$. Urine was collected via bladder catheter to determine urinary $\left[{ }^{3} \mathrm{H}\right]$ inulin excretion during this period.

\section{Analytical methods}

Tubular fluid inulin and total $\mathrm{CO}_{2}$ determinations were done as in previous studies from this laboratory $(8,12)$. Under the present experimental conditions the total $\mathrm{CO}_{2}$ concentration determined using microcalorimetry (15) was equated to the bicarbonate concentration in the sample. All tubular fluid bicarbonate concentrations were determined on the same day of the experiment using an 8-nl aliquot compared with an equivalent volume of a $10-\mathrm{mM} \mathrm{NaHCO} 3$ standard run concomitant with the samples. The standard generated a peak size of $20.4 \pm 1.2$ counts/pM and an SE of $0.40 \pm 0.05 \mathrm{mM}$ for all 28 experiments done for the study. Microcalorimetry was also used to determine total $\mathrm{CO}_{2}$ of anaerobically collected and centrifuged arterial plasma as well as urine collected under water and Hepes-equilibrated mineral oil. A bicarbonate standard of $25 \mathrm{mM}$ was used with the plasma and urine samples. 
Urine, plasma sodium, potassium, chloride and plasma protein concentrations were taken as described previously from this laboratory $(8,12)$.

\section{Calculations}

The SNGFR and whole kidney GFR were determined using the early distal SNGFR and the whole animal inulin clearance, respectively. The single nephron bicarbonate-filtered load was calculated using the early distal SNGFR multiplied by the plasma total $\mathrm{CO}_{2}\left(\mathrm{tCO}_{2}\right)$ corrected for plasma water and fór Donnan's equilibrium. A correction factor of 1.05 was used for the controls (16) and 1.06 was used for the alkalotic animals (4). Absolute bicarbonate reabsorption for the proximal tubule was determined as in previous studies from this laboratory $(8,12)$ by subtracting the bicarbonate-delivered load to the late proximal tubule from the single nephron filtered load. Late proximal bicarbonate-delivered load was calculated by multiplying the late proximal bicarbonate concentration determined from fluid obtained with minimal interruption of tubular flow (without on oil block) (see Micropuncture protocol) by the calculated late proximal flow rate. Late proximal flow rate was calculated by dividing the mean early distal SNGFR for that animal by the animal's mean inulin tubular fluid/ plasma ratio for the late proximal tubule. This calculated late proximal flow rate is a more accurate representation of the in situ flow rate because of the increase in proximal tubular flow elicited by discontinuation of loop of Henle fluid flow during proximal tubule fluid collections that use an oil block $(7,8)$. This calculation assumes maintenance of glomerular tubular balance for fractional fluid reabsorption with changes in proximal tubule flow rate. Other investigators have shown that changes in proximal tubule flow rate elicited by changes in SNGFR result in maintained fractional proximal tubule fluid reabsorption $(17,18)$. By contrast, fractional reabsorption for bicarbonate may not be maintained by the proximal tubule in response to increases in SNGFR (13). Therefore, comparison values for bicarbonate reabsorption by the proximal tubule were calculated using SNGFR, tubular bicarbonate concentration, and measured flow rate determined from late proximal fluid collected using an oil block as done by other investigators (4-6). Bicarbonate reabsorption for the loop segment was determined by subtracting the early distal bicarbonate delivery from the late proximal bicarbonate delivery. Bicarbonate reabsorption for the distal segment was calculated by subtracting the late distal bicarbonate delivery from the early distal bicarbonate-delivered load. Bicarbonate reabsorption for the distal segment was measured for only the 4-wk animals because reliable determinations of late distal flow rate could not be consistently done in the 1-wk alkalotic animals due to very slow flow. Fractional bicarbonate reabsorption for a given tubular segment was calculated by dividing the absolute reabsorption by the delivered load to that segment.

Inulin space (in milliliters) was calculated by dividing the retained inulin dose (administered dose minus urinary excretion) by plasma inulin concentration at time zero estimated by linear regression. The value was expressed as percent body weight.

Values are expressed as means $\pm \mathrm{SE}$. The results of the individual tubules were averaged for each animal for each puncture site. Statistical significance was determined using the $t$ test for unpaired observations.

\section{Results}

\section{Characterization of the model}

Animal growth data. There were no differences in body weights among regular chow-fed, control, and alkalotic animals at the start of the protocol $(177 \pm 4,175 \pm 5$, and $175 \pm 3 \mathrm{~g}$, respectively, $P=\mathrm{NS}), 1 \mathrm{wk}$ into the protocol $(207 \pm 4,203 \pm 5$, and $196 \pm 3 \mathrm{~g}$, respectively, $P=\mathrm{NS}$ ) or 4 wk into the protocol $(252 \pm 7,251 \pm 4$, and $253 \pm 3 \mathrm{~g}$, respectively, $P=\mathrm{NS})$. Left kidney weights of alkalotic $(n=6)$ and control $(n=6)$ animals micropunctured at 1 wk were not different $(0.981 \pm 0.017$ vs. $0.997 \pm 0.020 \mathrm{~g}$, respectively) and were not different from that of five animals with similar body weights fed standard rat chow $(1.006 \pm 0.015 \mathrm{~g}) .4 \mathrm{wk}$ into the protocol, left kidney weights of alkalotic animals were significantly greater than that of both control animals $(1.260 \pm 0.056$ vs. $1.061 \pm 0.028, P<0.006)$ and that of the comparison animals on standard chow $(1.083 \pm 0.022, P<0.03)$.

Electrolyte balance studies. Cumulative electrolyte balances for sodium, potassium, and chloride are shown in Fig. 1. In this figure, week 0 refers to the time just before the switch from the distilled $\mathrm{H}_{2} \mathrm{O}$ to the respective sodium salt drinking solution (see Methods). Alkalotic and control animals had similar negative balances for each measured electrolyte before the switch to the sodium salt drinking solution. At $1 \mathrm{wk}$, cumulative net balances for sodium were similar for the two groups but alkalotic vs. control balances for chloride $(-810 \pm 111$ vs. $2,631 \pm 617 \mu \mathrm{eq}$, respectively, $P<0.001)$ and

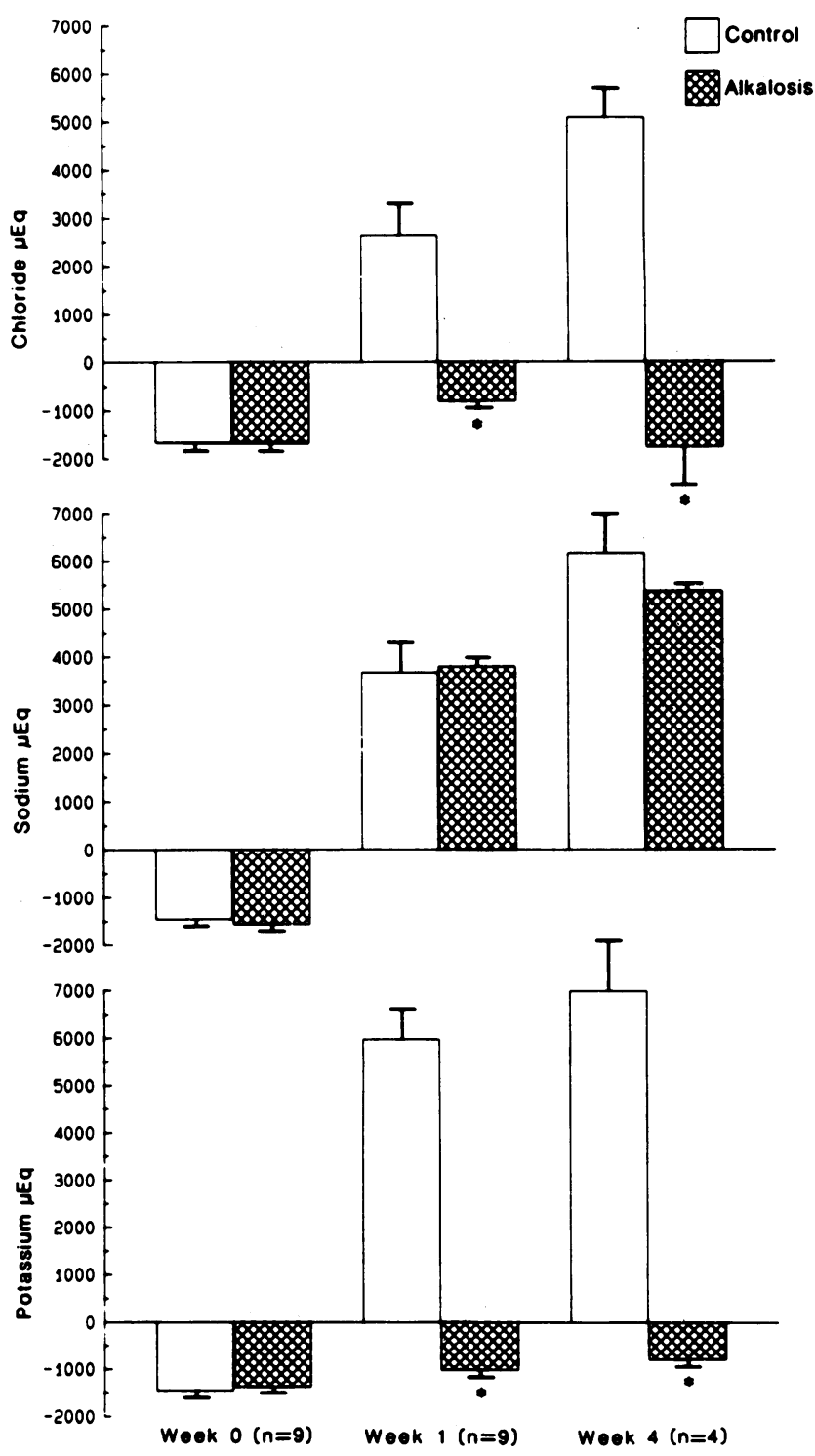

Figure 1. Cumulative whole animal electrolyte balances for sodium, potassium, and chloride for control and alkalotic animals. $n=$ number of animals; ${ }^{*} P<0.05$ vs. respective control. 


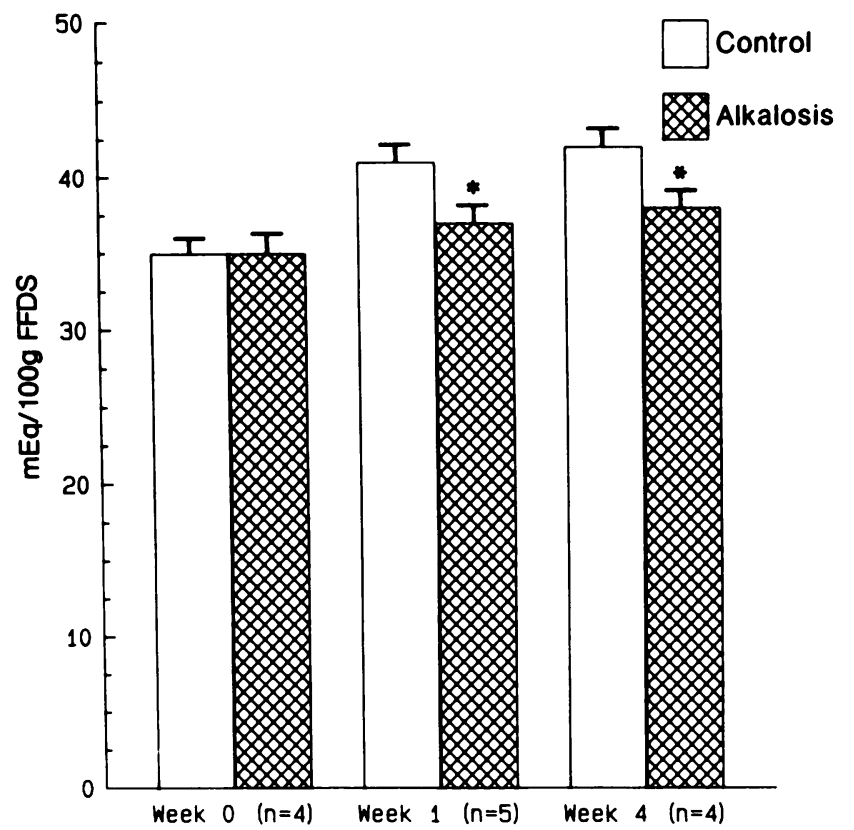

Figure 2. Muscle potassium concentration per fat-free dry solid (FFDS) weight. ${ }^{*} P<0.05$ vs. respective control.

for potassium $(-1,017 \pm 162$ vs. $5,881 \pm 610 \mu$ eq, respectively, $P$ $<0.001)$ were significantly lower. This pattern was maintained at $4 \mathrm{wk}$ for the alkalotic animals showing similar sodium balances but persistently negative balances for chloride $(-1,767 \pm 793$ vs. $5,077 \pm 597 \mu \mathrm{eq}$, respectively, $P<0.001)$ and for potassium $(-793 \pm 143$ vs. $7,193 \pm 1,021 \mu \mathrm{eq}$, respectively, $P$ $<0.001)$. There were no differences in chloride nor potassium balance comparing the alkalotic animals at the 1- and 4-wk time intervals. By 1 wk into the protocol, the alkalotic animals had not yet ingested enough chloride from their low electrolyte diet (14 $\mu$ eq chloride/g diet) to make up their initial chloride deficit. These animals maintained a chloride-free urine. By 4 wk, cumulative dietary chloride ingestion by the alkalotic animals was in excess of their initial deficit but these animals remained in negative chloride balance due to a small but persistent urinary chloride excretion beginning $\sim 2 \mathrm{wk}$ into the protocol. These alkalotic animals had persistently high urinary potassium excretion despite its provision as nonchloride salts in the drinking solution and in the diet.

Muscle potassium determination. Muscle potassium concentrations for the indicated time intervals are depicted in Fig. 2. Both the alkalotic and control animals had similar muscle potassium concentrations $48 \mathrm{~h}$ into the induction protocol ( $34 \pm 1.5$ vs. $34 \pm 1.1 \mathrm{meq} / 100 \mathrm{~g}$ FFDS, respectively) that were both significantly lower that the values obtained from five animals of similar weight which had received standard rat chow and no furosemide $(41 \pm 0.9 \mathrm{meq} / 100 \mathrm{~g}$ FFDS, $P<0.004$ vs. both alkalotic and control). At $1 \mathrm{wk}$, control muscle potassium concentration ( $40 \pm 1.2 \mathrm{meq} / 100 \mathrm{~g}$ FFDS) was comparable to that of standard chow animals but that of the alkalotic animals remained lower than that for both the standard chow and respective control animals $(35 \pm 1.3 \mathrm{meq} / 100 \mathrm{~g}$ FFDS, $P$ $<0.03$ vs. both standard chow and respective control). At 4 wk, this pattern was maintained with the muscle potassium of the control animals being comparable to that of standard chow animals but that for the alkalotic animals being lower $(36 \pm 1.1$ vs. $41 \pm 1.4 \mathrm{meq} / 100 \mathrm{~g}$ FFDS, $P<0.03$ ).

Inulin space determinations. Inulin spaces expressed as percent body weight were not different for alkalotic compared with control animals at 1 wk $(20.0 \pm 0.9$ vs. $19.9 \pm 0.4 \%)$ or at 4 wk $(21.8 \pm 1.3$ vs. $21.8 \pm 2.1 \%)$. To determine the sensitivity of the method (see Methods) to recognize a deficit in extracellular fluid volume, inulin space determinations were done in 10 animals of similar body weights $48 \mathrm{~h}$ into the induction protocol before being given salt replacement in their drinking water. These animals had deficits in sodium, potassium, and chloride similar to that of the alkalotic and control animals at week zero depicted in Fig. 1. These clearly volume-depleted animals had a smaller measured inulin space compared with that of the above control animals $(17.1 \pm 1.1$ vs. $19.9 \pm 0.4 \%, P<0.03)$.

\section{Arterial blood composition and pressure}

Table I depicts arterial plasma electrolyte and protein concentrations as well as blood hematocrits at the 1- and 4-wk time intervals. These values were obtained from femoral arterial blood of the micropunctured animals immediately before initiation of micropuncture and did not change significantly during the micropuncture period. Sodium concentration did not differ between groups. Plasma potassium concentration was lower in the alkalotic animals at both time intervals, reaching statistical significance only at the $1-w k$ interval. Alkalotic

Table I. Arterial Blood Composition

\begin{tabular}{|c|c|c|c|c|c|c|}
\hline & $\mathrm{Na}^{+}$ & $\mathrm{K}^{+}$ & $\mathrm{Cl}^{-}$ & $\mathrm{tCO}_{2}$ & Hct & Protein \\
\hline & \multicolumn{4}{|c|}{ meq/liter } & $\%$ & $g / d l$ \\
\hline & \multicolumn{6}{|c|}{$1 \mathrm{wk}$} \\
\hline Control $(n=6)$ & $149 \pm 2$ & $3.9 \pm 0.2$ & $113 \pm 2$ & $24.1 \pm 1.7$ & $47.5 \pm 0.5$ & $4.0 \pm 0.1$ \\
\hline \multirow[t]{2}{*}{ Alkalosis $(n=6)$} & $148 \pm 2$ & $2.8 \pm 0.2^{*}$ & $97 \pm 3^{*}$ & $41.5 \pm 2.1^{*}$ & $47.1 \pm 0.9$ & $4.1 \pm 0.1$ \\
\hline & \multicolumn{6}{|c|}{4 wk } \\
\hline Control $(n=8)$ & $148 \pm 2$ & $4.0 \pm 0.2$ & $114 \pm 1$ & $23.7 \pm 1.2$ & $47.8 \pm 0.7$ & $4.1 \pm 0.1$ \\
\hline Alkalosis $(n=8)$ & $146 \pm 1$ & $3.4 \pm 0.2$ & $102 \pm 3^{*}$ & $36.2 \pm 1.3^{*}$ & $48.2 \pm 0.8$ & $4.0 \pm 0.1$ \\
\hline
\end{tabular}

Values are mean $\pm \mathrm{SE}, n=$ number of animals. ${ }^{*} P<0.05$ vs. respective control. 
Table II. GFR and Urinary Anion Excretion

\begin{tabular}{|c|c|c|c|}
\hline & Whole animal GFR & $\mathrm{U}_{\mathrm{c}} \mathrm{V}$ & $\mathrm{U}_{\mathrm{tCO}_{2}} \mathrm{~V}$ \\
\hline & $\mu l / m i n$ & neq/min & neq/min \\
\hline & & $1 \mathrm{wk}$ & \\
\hline Control $(n=6)$ & $1,986 \pm 214$ & $2,025 \pm 376$ & $61 \pm 10$ \\
\hline \multirow[t]{2}{*}{ Alkalosis $(n=6)$} & $1,354 \pm 153^{*}$ & $285 \pm 49^{*}$ & $149 \pm 18^{*}$ \\
\hline & & $4 \mathrm{wk}$ & \\
\hline Control $(n=8)$ & $1,758 \pm 145$ & $2,243 \pm 290$ & $47 \pm 29$ \\
\hline Alkalosis $(n=8)$ & $2,352 \pm 302$ & $449 \pm 57^{*}$ & $50 \pm 7^{\ddagger}$ \\
\hline
\end{tabular}

Values are means $\pm \mathrm{SE}, n=$ number of animals.

* $P<0.05$ vs. respective control.

${ }^{\ddagger} P<0.05$ vs. respective $1-w k$ value.

compared with control animals were hypochloremic and had higher concentrations for plasma $\mathrm{tCO}_{2}$ at both time intervals. These chloride and $\mathrm{tCO}_{2}$ values were not different when comparing respective 1- and 4-wk values for either the alkalotic or control animals. Hematocrit and plasma protein concentrations were not different between groups at either time interval. Mean femoral arterial blood pressures were not different in alkalotic vs. control animals at $1 \mathrm{wk}(126 \pm 5 \mathrm{vs} .125 \pm 5 \mathrm{mmHg})$ or $4 \mathrm{wk}(131 \pm 2$ vs. $128 \pm 2 \mathrm{mmHg})$.

\section{GFR and urinary data}

Whole animal glomerular filtration rates as well as urinary anion excretion (from both kidneys) determined during micropuncture are depicted in Table II. The GFR of alkalotic animals was lower at 1 wk but was not statistically different at the 4-wk interval. Comparing respective 4- and 1-wk values for GFR within groups, that for the alkalotic animals at 4 wk was greater but these respective values were not different for control animals. Urinary chloride excretion was lower in alkalotic animals at both time intervals. There was no difference comparing 1- and 4-wk values for either group. Urinary bicarbonate excretion of alkalotic animals was higher at 1 wk but was not different at $4 \mathrm{wk}$. The alkalotic animals had lower urinary bicarbonate excretion at $4 \mathrm{wk}$ compared with the respective 1 -wk value but these respective values were not different for control animals.

\section{Tubular bicarbonate reabsorption}

Proximal tubule bicarbonate reabsorption was assessed using two different methods for collecting the proximal tubule fluid (see Methods). Table III depicts data obtained from late proximal tubule fluid collected with ("blocked") and without ("noblock") an oil block. The SNGFR reported for the no-block collection is that determined at the early distal tubule for that group of animals. This early distal value was significantly lower than that determined from quantitative (blocked) collections from the proximal tubule of both alkalotic and control animals at each time interval. The late proximal flow rate for the no-block collections was calculated using the early distal SNGFR and the inulin tubular-fluid-to-plasma ratio measured at the late proximal tubule (see Methods). This calculated value was lower than the respective measured value for all groups, reaching statistical significance for the 4-wk control animals. Inulin tubular-fluid-to-plasma ratios were not differ-
Table III. Comparison of Proximal Tubule Data Obtained with and without an Oil Block

\begin{tabular}{|c|c|c|c|c|}
\hline & SNGFR & Flow rate & $(\mathrm{TF} / \mathrm{P})_{\mathrm{I}}$ & {$\left[\mathrm{HCO}_{3}\right]$} \\
\hline \multicolumn{4}{|c|}{$n l / m i n$} & meq/liter \\
\hline & \multicolumn{4}{|c|}{ Control $(1 \mathrm{wk})(n=6)$} \\
\hline Blocked & $39.5 \pm 2.9$ & $20.2 \pm 2.7$ & $2.0 \pm 0.2$ & $10.1 \pm 1.2$ \\
\hline \multirow[t]{2}{*}{ No block } & $27.6 \pm 1.2^{\ddagger}$ & $14.1 \pm 1.8$ & $2.0 \pm 0.1$ & $7.9 \pm 1.0$ \\
\hline & \multicolumn{4}{|c|}{ Alkalosis $(1 \mathrm{wk})(n=6)$} \\
\hline Blocked & $31.7 \pm 2.4$ & $14.3 \pm 1.5$ & $2.3 \pm 0.2$ & $19.1 \pm 1.5^{*}$ \\
\hline \multirow[t]{2}{*}{ No block } & $22.2 \pm 1.7^{* \ddagger}$ & $10.2 \pm 1.2$ & $2.2 \pm 0.2$ & $14.0 \pm 1.2^{* \ddagger}$ \\
\hline & \multicolumn{4}{|c|}{ Control (4 wk) $(n=8)$} \\
\hline Blocked & $38.1 \pm 2.7$ & $17.5 \pm 1.8$ & $2.2 \pm 0.2$ & $9.8 \pm 1.1$ \\
\hline \multirow[t]{2}{*}{ No block } & $26.1 \pm 1.9^{\ddagger}$ & $11.5 \pm 1.7^{\ddagger}$ & $2.3 \pm 0.2$ & $8.1 \pm 0.6$ \\
\hline & \multicolumn{4}{|c|}{ Alkalosis (4 wk) $(n=6)$} \\
\hline Blocked & $42.6 \pm 2.1$ & $13.2 \pm 1.0$ & $3.3 \pm 0.3^{*}$ & $15.2 \pm 1.3^{*}$ \\
\hline No block & $33.6 \pm 1.1^{* \neq}$ & $10.8 \pm 1.4$ & $3.1 \pm 0.2^{*}$ & $11.0 \pm 1.0^{\ddagger}$ \\
\hline
\end{tabular}

Values are means \pm SE, $n=$ number of animals.

$* P<0.05$ vs. control.

${ }^{\ddagger} P<0.05$ vs. blocked value.

The no block SNGFR is that determined at the early distal tubule.

ent when comparing the two collection methods. These ratios were higher in the alkalotic animals studied at $4 \mathrm{wk}$ but not at $1 \mathrm{wk}$. Tubular bicarbonate concentrations at the late proximal tubule were lower with the no-block method for all groups, reaching statistical significance for both alkalotic groups.

Table IV depicts bicarbonate reabsorptive data for the

Table IV. Comparison of Bicarbonate Reabsorption Data for the Proximal Tubule Assessed with and without an Oil Block

\begin{tabular}{|c|c|c|c|c|}
\hline & Filtered load & $\begin{array}{l}\text { Delivered } \\
\text { load }\end{array}$ & $\begin{array}{l}\text { Absolute } \\
\text { reabsorption }\end{array}$ & $\begin{array}{l}\text { Fractional } \\
\text { reabsorption }\end{array}$ \\
\hline & & $p M / \min$ & & $\%$ \\
\hline & \multicolumn{4}{|c|}{ Control (1 wk) $(n=6)$} \\
\hline Blocked & $1,044 \pm 124$ & $207 \pm 17$ & $836 \pm 115$ & $81 \pm 3$ \\
\hline \multirow[t]{2}{*}{ No block } & $721 \pm 69^{\ddagger}$ & $114 \pm 12^{\ddagger}$ & $607 \pm 57$ & $84 \pm 2$ \\
\hline & \multicolumn{4}{|c|}{ Alkalosis $(1 \mathrm{wk})(n=6)$} \\
\hline Blocked & $1,452 \pm 171$ & $274 \pm 19 *$ & $1,177 \pm 148$ & $81 \pm 3$ \\
\hline \multirow[t]{2}{*}{ No block } & $1,004 \pm 105^{* \ddagger}$ & $141 \pm 15^{\ddagger}$ & $863 \pm 90^{*}$ & $86 \pm 2$ \\
\hline & \multicolumn{4}{|c|}{ Control $(4$ wk) $(n=8)$} \\
\hline Blocked & $989 \pm 109$ & $170 \pm 16$ & $819 \pm 79$ & $83 \pm 2$ \\
\hline \multirow[t]{2}{*}{ No block } & $672 \pm 71^{\ddagger}$ & $95 \pm 10^{\ddagger}$ & $577 \pm 68^{\ddagger}$ & $86 \pm 2$ \\
\hline & \multicolumn{4}{|c|}{ Alkalosis $(4 \mathrm{wk})(n=6)$} \\
\hline Blocked & $1,701 \pm 195^{*}$ & $198 \pm 14$ & $1,502 \pm 121^{*}$ & $88 \pm 2$ \\
\hline No block & $1,374 \pm 82^{*}$ & $117 \pm 5^{\ddagger}$ & $1,257 \pm 80^{*}$ & $91 \pm 2$ \\
\hline
\end{tabular}

\footnotetext{
Values are means $\pm \mathrm{SE}, n=$ number of animals.

$* P<0.05$ vs. control.

${ }^{\ddagger} P<0.05$ vs. blocked value.
} 
proximal tubule using the data obtained from the two collection methods. In general, the no-block technique yielded absolute values that were lower than those obtained when an oil block was used, whereas fractional reabsorption did not differ so dramatically. Filtered load and absolute reabsorption of bicarbonate were significantly higher in the alkalotic animals at both time intervals when comparing values determined using the no-block technique. When an oil block was used, this relationship was statistically significant for only the animals studied at $4 \mathrm{wk}$. Given that the no-block technique yields less stimulation of the tubuloglomerular feedback response (see Methods) and thus more closely reflects in situ conditions, this method was chosen to assess proximal tubule bicarbonate reabsorption.

When examination of bicarbonate reabsorption by the proximal tubule is limited to the no no-block technique, Table IV shows that bicarbonate filtered load and absolute reabsorption were higher in the alkalotic animals studied at each time interval. Comparing the 4- and 1-wk values within groups, the alkalotic animals had greater values for both bicarbonate filtered load $(P<0.02)$ and absolute reabsorption $(P<0.03)$ at 4 wk, but these respective values were not different for control animals. Fractional bicarbonate reabsorption for the proximal tubule was not different between groups. Fig. 3 (top) shows that the slopes of the linear regression comparing filtered load (delivered load) to absolute reabsorption of bicarbonate for the proximal tubule were not different between alkalotic and control animals studied at 1 wk $(0.87 \pm 0.03$ vs. $0.85 \pm 0.03$, respectively, $P=\mathrm{NS})$ and at $4 \mathrm{wk}(0.97 \pm 0.03$ vs. $0.87 \pm 0.04$, respec-

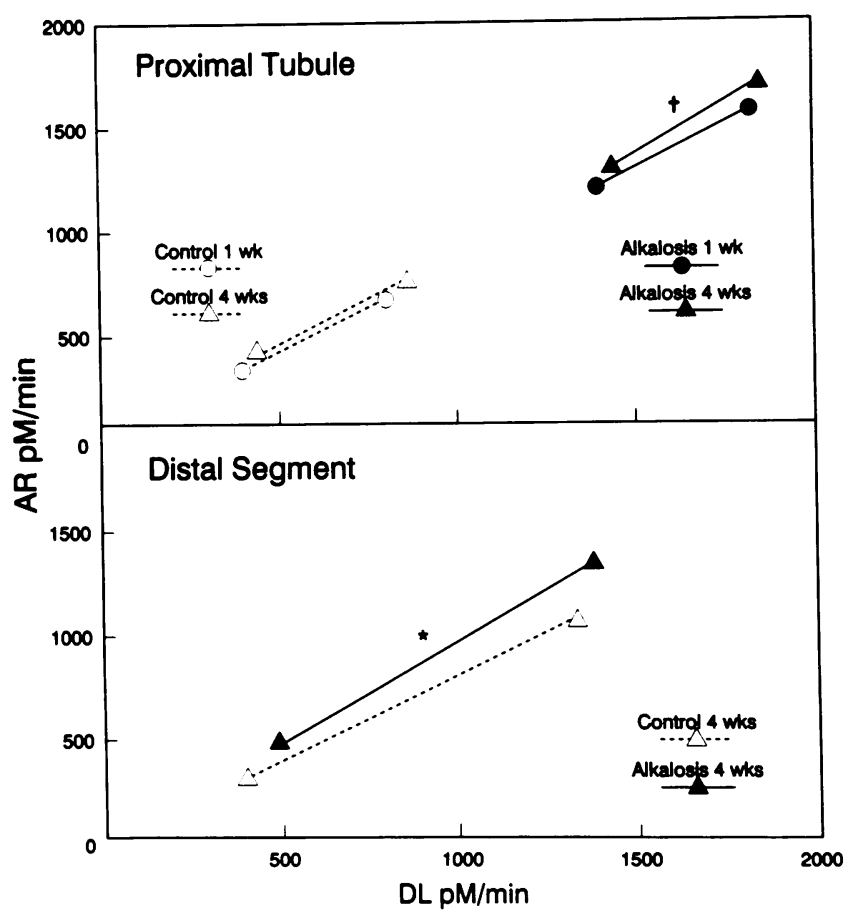

Figure 3. Linear regression for absolute reabsorption $(A R)$ vs. delivered load $(D L)$ of bicarbonate to the proximal tubule and for absolute reabsorption vs. delivered load of bicarbonate to the distal segment. The dashed lines and open symbols represent control animals, the solid lines with closed symbols represent alkalotic animals. The circles represent animals studied at $1 \mathrm{wk}$, the triangles animals studied at $4 \mathrm{wk} .{ }^{*} P<0.05$ for slope of regression vs. respective control; ${ }^{+} P<0.05$ for slope of regression vs. respective 1-wk value. tively, $P=$ NS). Comparing the 4- and 1-wk slopes within groups, the alkalotic animals had a higher value at 4 wk $(P$ $<0.04$ ), but these respective values were not different for control animals.

Bicarbonate reabsorption parameters for the loop segment are depicted in Fig. 4. There was no difference in delivered load to or absolute reabsorption from this nephron segment between groups studied at either time interval.

Fig. 5 depicts bicarbonate reabsorption parameters for the distal segment. This figure shows data for only the 4-wk animals because accurate late distal collections could not be obtained in the alkalotic animals studied at one week (see Methods). Alkalotic animals had higher bicarbonate delivery to ( $44 \pm 3$ vs. $26 \pm 3 \mathrm{pM} / \mathrm{min}, P<0.002)$ as well as higher absolute reabsorption from this segment $(35 \pm 3$ vs. $19 \pm 3 \mathrm{pM} / \mathrm{min}$, $P<0.004)$ than did the control animals. There was no significant difference in fractional bicarbonate reabsorption by the distal segment between groups. Fig. 3 (bottom) shows that the slope of the linear regression comparing delivered load to absolute reabsorption of bicarbonate from the distal segment was greater in the alkalotic animals $(0.98 \pm 0.03$ vs. $0.81 \pm 0.03, P$ $<0.003$ ).

Fig. 6 depicts the tubular fluid/plasma concentration ratios $(\mathrm{TF} / \mathrm{P})$ for inulin and bicarbonate at the three puncture sites for the alkalotic and control animals. Late distal values are not depicted for the animals studied at $1 \mathrm{wk}$ for the reasons stated above. The inulin TF/P ratio at the late proximal tubule was higher in the alkalotic animals studied at $4 \mathrm{wk}$ but was the same in animals studied at $1 \mathrm{wk}$. Despite this difference in fractional fluid reabsorption by the proximal tubule of alkalotic animals studied at $4 \mathrm{wk}$, the bicarbonate $\mathrm{TF} / \mathrm{P}$ ratios at the late proximal tubule were not different between groups studied at both time intervals. Early distal and late distal inulin TF/P ratios were not different when comparing respective ratios for each site between alkalotic and control animals. Bicarbonate $\mathrm{TF} / \mathrm{P}$ ratios at the early distal tubule were not different between groups but those at the late distal site were significantly lower in the alkalotic animals $(0.10 \pm 0.01$ vs. $0.16 \pm 0.01, P$ $<0.006)$. The lower bicarbonate TF/P ratio at the late distal tubule of alkalotic animals was not due to lower fluid flow rates in this segment of alkalotic compared with control animals at either the early distal $(7.3 \pm 1.2$ vs. $5.5 \pm 0.6 \mathrm{nl} / \mathrm{min}$, respectively, $P=\mathrm{NS})$ or late distal $(2.3 \pm 0.3 \mathrm{vs} .1 .8 \pm 0.2 \mathrm{nl} /$ min, respectively, $P=\mathrm{NS}$ ) sites.

Surface length of micropunctured tubules were determined using latex injections (see Methods). The proximal tubule length measured was from the site of puncture to the glomerulus. The surface distal tubule length measured was between early and late distal puncture sites. At the 1-wk time interval, alkalotic and control animals had similar lengths for both proximal $(4.9 \pm 0.3$ vs. $4.7 \pm 0.3 \mathrm{~mm})$ and distal $(0.9 \pm 0.1$ vs. $1.0 \pm 0.1 \mathrm{~mm})$ tubules. For the 4-wk interval, proximal tubule length was greater for alkalotic animals $(6.1 \pm 0.3$ vs. $5.1 \pm 0.2$ $\mathrm{mm}, P<0.02$ ) but distal tubule length was not different $(1.1 \pm 0.02$ vs. $1.0 \pm 0.1 \mathrm{~mm})$.

\section{Discussion}

This free-flow micropuncture study demonstrates that increased bicarbonate reabsorption by both the proximal and distal nephron contributes to maintaining the alkalosis in a 


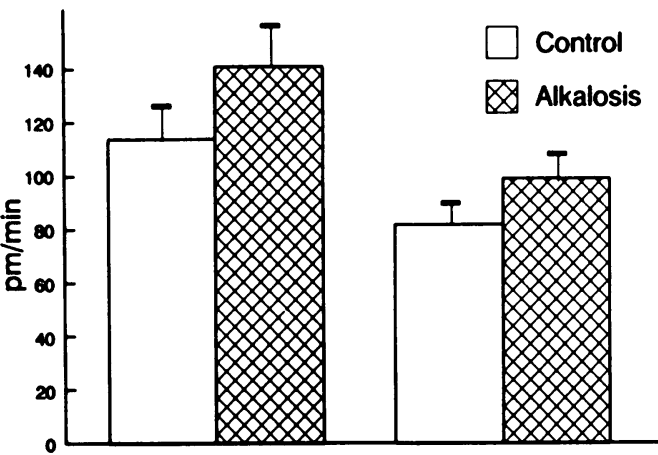

DL

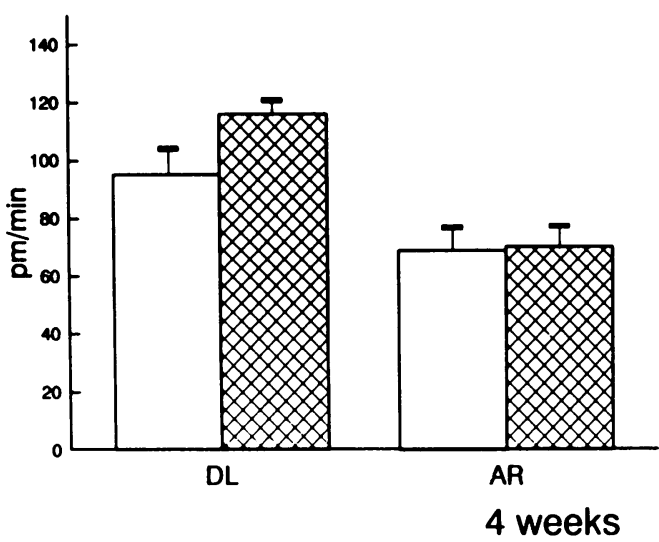

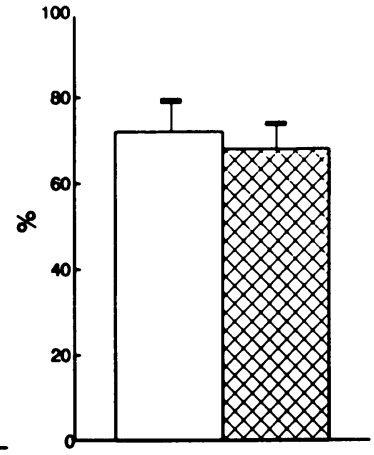

FR

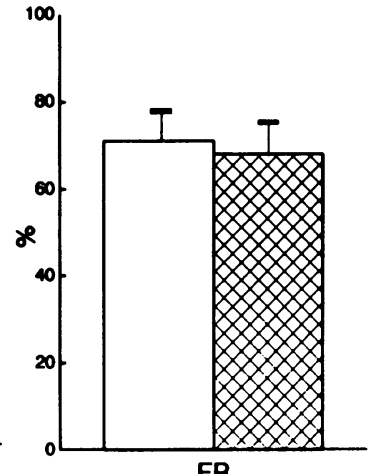

FR

Figure 4. Loop segment delivered load $(D L)$, absolute reabsorption $(A R)$, and fractional reabsorption $(F R)$ of bicarbonate. For $1 \mathrm{wk}, n=6$ for both control and alkalotic animals. For 4 wk, $n=8$ for control, and $n=6$ for the alkalotic animals.

chloride-deplete model of this disorder in rats. The participation of the distal nephron in maintaining this acid-base disorder must be added to that previously described for the proximal tubule $(1,2,6)$ and for GFR (4). The increased reabsorption by the proximal tubule was associated with similar slopes of the linear regression between absolute reabsorption and filtered load as well as similar values for the bicarbonate TF/P ratios comparing alkalotic and control animals. These data suggest that the increased reabsorption of bicarbonate by the proximal tubule was in response to the increased filtered load. By contrast, the increased reabsorption by the distal segment of the alkalotic animals was associated with a greater slope for

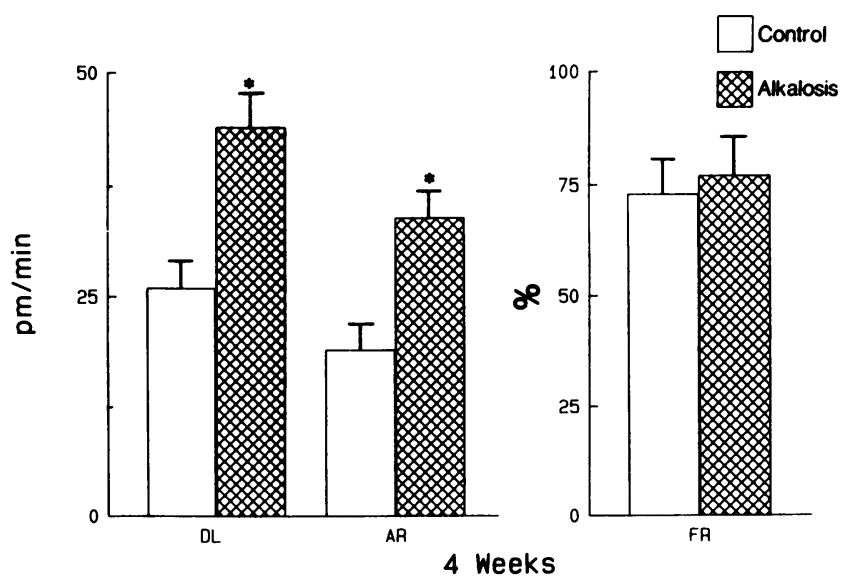

Figure 5. Distal segment delivered load, absolute reabsorption, and fractional reabsorption of bicarbonate. $n=6$ for both control and alkalotic animals. ${ }^{*} P<0.05$ vs. respective control. the linear regression between absolute reabsorption and delivered load as well as a lower value for the bicarbonate TF/P ratio at the late distal tubule. These data suggest that the increased reabsorption of bicarbonate by the distal segment was
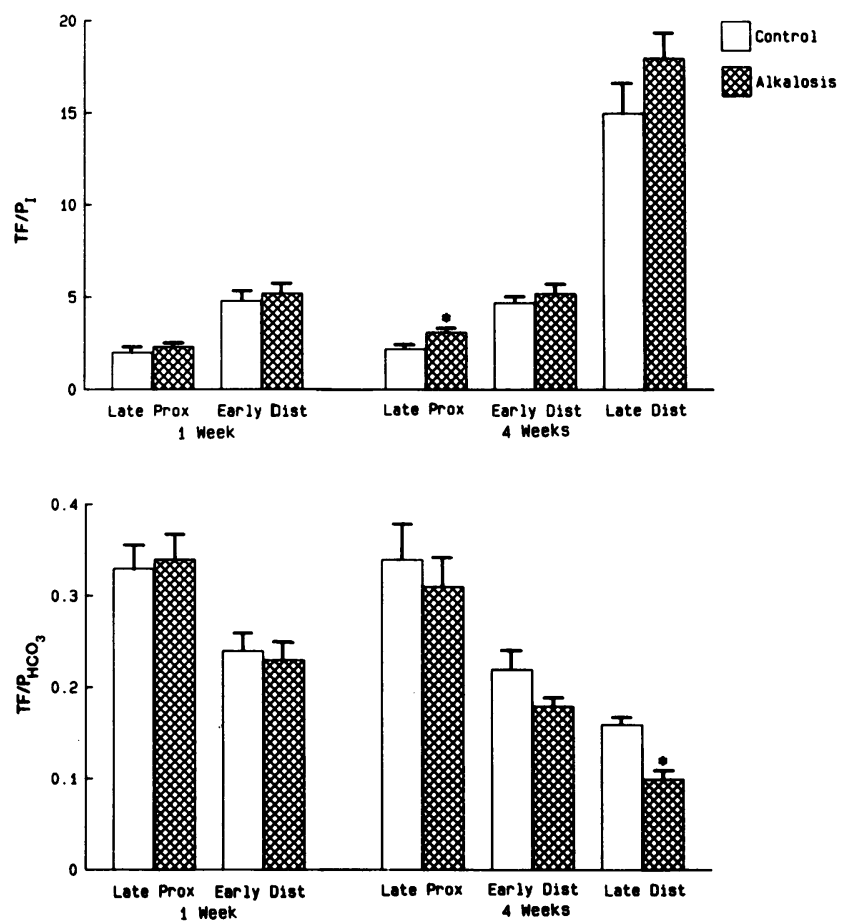

Figure 6. TF/P ratio for inulin $(I)$ and bicarbonate at the indicated puncture sites. ${ }^{*} P<0.05$ vs. respective control value. 
due to stimulated bicarbonate reabsorption in addition to the increased delivered load. This study thus suggests that maintenance of chloride-deplete alkalosis involves stimulated bicarbonate reabsorption in the distal nephron but reabsorption in the proximal tubule, which is increased primarily in response to an increased filtered load of bicarbonate.

In this study, bicarbonate reabsorption by the proximal tubule was assessed using quantitative fluid collections from this segment using an oil block and alternatively from proximal tubule collections done without an oil block. Previous free-flow micropuncture studies of chloride-deplete metabolic alkalosis used the oil-block method to assess bicarbonate reabsorption in the proximal tubule (1-6). The advantage of this method is that all the components used to determine bicarbonate reabsorption in the proximal tubule (SNGFR, late proximal flow rate, and tubular bicarbonate concentration) can be measured directly. This allows for determination of a unique value for bicarbonate reabsorption by that tubule being punctured. The disadvantage is that complete interruption of fluid flow to the loop of Henle maximally stimulates the TGF response leading to increased $\operatorname{SNGFR}(7,8)$ with its resultant artifactual influences. This effect is evident from the data in Table III, which show greater SNGFR when measured in the proximal than in the distal nephron. Other investigators have shown that increases in SNGFR (13) and increases in proximal tubule flow rate with in vivo microperfusion (19) can lead to greater bicarbonate concentrations in the tubular fluid at the terminal proximal tubule. This effect is also evident in Table III but is disproportionately greater in the alkalotic animals than in their respective controls. Other investigators reported similar findings in that the described effect was most evident in normal compared with acidotic animals (16) and in perfusates of higher bicarbonate concentrations (19). The advantage of the no-block technique (see Methods) is that it reduces stimulation of the TGF response and thereby yields measurements for the described components which are closer to those existing in situ. The disadvantage of this method is that the SNGFR used is a mean value determined from random nephrons and this value was used to calculate the late proximal flow rate (see Methods). Therefore, this method yielded a measurement for bicarbonate reabsorption in the proximal tubule, which was a mean value for the kidney rather than a specific value for the proximal tubule being punctured. Nevertheless, the no-block method reduces the artifactual influences described above. For this reason, the no-block method was chosen to assess bicarbonate reabsorption of the proximal tubule in this study. Despite the stated advantage of the no-block technique, Table IV shows that qualitative comparisons of bicarbonate transport parameters of the proximal tubule between alkalotic and control animals were generally but not statistically similar when using the two collection methods.

The data of this study demonstrate that both the proximal and the distal nephron contribute to the maintenance of chloride-deplete metabolic alkalosis but suggest that they do so by different mechanisms. Bicarbonate reabsorption in the distal segment appears to be augmented due to a primary stimulation of bicarbonate reabsorption in this nephron segment. By contrast, the data suggest that bicarbonate reabsorption by the proximal tubule is increased secondarily in response to an increased filtered load, which occurs in part because of stimulated bicarbonate reabsorption by the distal segment.
The etiology for the augmented bicarbonate reabsorption in the distal nephron of the alkalotic animals is not entirely clear but may relate to the demonstrated potassium depletion. Prolonged potassium restriction in rats has been associated with increased fractional reabsorption of bicarbonate and lower bicarbonate TF/P ratios in the distal segments of these animals (20). The alkalotic animals of this study showed a weak inverse correlation between plasma potassium concentration and distal segment bicarbonate reabsorption ( $r$ $=-0.78, P=0.067)$. There was no such correlation for control animals $(r=0.54, P=0.21)$. Thus, the potassium depletion of the alkalotic animals may be responsible for the augmented reabsorption seen in this segment.

Load-dependent bicarbonate reabsorption by the proximal tubule was maintained in the alkalotic animals despite the presence of alkalosis. Although the longer proximal tubule of the alkalotic animals studied at $4 \mathrm{wk}$ may have contributed to the increase in bicarbonate reabsorption seen at this interval, the similar proximal tubule lengths of the animals studied at 1 wk suggests that increased tubule length does not contribute to the increased bicarbonate reabsorption seen in these animals. Investigators studying bicarbonate reabsorption of proximal tubule segments have shown that the early proximal tubule has the greatest capacity for bicarbonate reabsorption (21) and that the load dependence of this early segment is not diminished by systemic alkalosis (13). Because the majority of bicarbonate reabsorption by the proximal tubule occurs in this early segment $(13,21)$, the load dependence found for this segment in this study is consistent with the response of the early proximal tubule to an increase in the filtered load of bicarbonate. The present study also showed that the slope of the reabsorption vs. filtered load relationship was greater for the alkalotic animals studied at $4 \mathrm{wk}$ than in those studied at $1 \mathrm{wk}$. This greater slope was associated with similar bicarbonate $T F / P$ ratios. These data are compatible with the longer proximal tubule of the 4-wk alkalotic animals (see Results) allowing for more bicarbonate reabsorption to occur with a given filtered load. Given that the load dependence of these longer proximal tubules was not diminished in the face of alkalosis, the added proximal tubule epithelium was likely characteristic of the early portion.

Other factors may have contributed to the increased bicarbonate reabsorption by the proximal tubule of the alkalotic animals. Potassium depletion has been associated with intracellular acidosis (22) and hyperpolarization (23), both of which may stimulate $\mathrm{Na}^{+}-\mathrm{H}^{+}$exchange. Chronic hypokalemia has been associated with increased bicarbonate reabsorption by the proximal tubule (24). The stimulated bicarbonate reabsorption would be theoretically counterbalanced by the resulting alkalosis, at least in the latter portions of the proximal tubule $(13,25)$. Such a system may permit load-dependent bicarbonate reabsorption to occur. Chloride depletion has been implicated in the etiology of metabolic alkalosis $(26,27)$ and was present in the alkalotic animals studied at both time intervals. There was a strong inverse correlation between serum chloride concentration and absolute bicarbonate reabsorption by the proximal tubules of the alkalotic animals studied at $1 \mathrm{wk}(r=-0.87, P=0.019)$ and at $4 \mathrm{wk}(r=-0.91, P$ $=0.012)$; there was no such correlation in control animals $(r$ $=0.3, P=0.4$ for $1 \mathrm{wk} ; r=0.2, P=0.5$ for $4 \mathrm{wk}$ ). Whether this relationship was specific for plasma chloride concentration or for some factor(s) associated with hypochloremia could not be 
determined. Finally, some investigators have implicated volume depletion as contributing to the increased tubular bicarbonate reabsorption seen with chloride-deplete metabolic alkalosis $(4,9)$. The alkalotic animals of this study did not appear to have lower ECF volumes than control, as supported by similar values for inulin space, sodium balance, arterial blood hematocrit and plasma protein concentration, blood pressure, and body weight. Though subtle degrees of volume depletion may not have been detected by the methods used, dramatic differences in ECF did not account for the differences in bicarbonate reabsorption between groups.

Comparison with previous studies. This study extends our knowledge as to how chloride-deplete metabolic alkalosis is maintained. First, this study shows that the distal nephron contributes to the maintenance of the alkalosis by means of stimulated bicarbonate reabsorption. This nephron segment has received relatively little attention in previous micropuncture studies of this acid-base disorder. In addition, this study shows that the increase in bicarbonate reabsorption seen in the proximal tubule is not dependent on increased length of this nephron segment or on prolonged ( $>1 \mathrm{wk}$ ) exposure to high filtered load of bicarbonate. Earlier studies had suggested that these two features might be prerequisites for the increase in bicarbonate reabsorption by the proximal tubule to occur (6). Finally, characterization of the model suggests that the increased tubular reabsorption may relate to depletion of potassium and/or chloride but does not appear to be due to substantial depletion of ECF volume. In agreement with previous studies, the data of this study demonstrate higher bicarbonate reabsorption in the proximal tubule of alkalotic animals (1, 2, 6), load dependence for proximal bicarbonate reabsorption that was comparable to that of control animals (6), lower GFR for alkalotic animals studied soon after induction $(4,5)$, higher SNGFR of animals with prolonged alkalosis (6), negative potassium balance for alkalotic animals (4), and similar inulin spaces for alkalotic and control animals (3). The major contrast between the data of this study and previous micropuncture studies is that an earlier micropuncture study demonstrated that alkalotic animals studied at 11-14 d had nephron filtered load of bicarbonate that was comparable to that of control because of a reciprocal reduction of GFR (4). As a result, bicarbonate reabsorption by the proximal tubule was also comparable to control. This study showed greater nephron filtered load of bicarbonate for alkalotic animals studied at both time intervals despite the reduced GFR of the animals studied at $1 \mathrm{wk}$. This increased filtered load was associated with increased bicarbonate reabsorption in the proximal tubule. Because oil blocks were used for proximal tubule collections in the previous study, stimulation of the TGF response and its described consequences may account in part for the differences between these two studies. In support of this, Table IV shows the bicarbonate filtered load and proximal reabsorption were not different between the alkalotic and control animals studied at one week when using an oil block. By contrast, those collections done without an oil block showed the differences which have been described. Additional differences between this and the earlier study include differences in the alkalosis induction protocol (the earlier study used dietary $\mathrm{Na}_{2} \mathrm{SO}_{4}$ and mineralocorticoid injection and no furosemide), the time after induction at which the animals were studied, more severe potassium depletion of the alkalotic animals of the earlier study, and the use of retrospective controls by the previous study.

In summary, this study demonstrates that increased bicarbonate reabsorption by both the proximal and the distal nephron contributes to the maintenance of chloride-deplete metabolic alkalosis in rats. The data suggest that bicarbonate reabsorption is stimulated in the distal nephron, whereas the increased reabsorption in the proximal nephron occurs as a result of increased filtered load. This study suggests a mechanism for maintenance of chloride-deplete metabolic alkalosis, which includes primary stimulation of bicarbonate reabsorption in the distal nephron and load-dependent bicarbonate reabsorption in the proximal tubule.

\section{Acknowledgments}

We are grateful to Debby S. Verrett for typing the manuscript, to Ernest Pace for technical assistance, and to Dr. Horacio J. Adrogue for reviewing the manuscript.

This work was supported by funds from the Merit Review Program of the Veterans Administration and from the United States Public Health Services grant RR-05425.

\section{References}

1. Bank, N., and H. S. Aynedjian. 1965. A micropuncture study of renal bicarbonate and chloride reabsorption in hypokalemic alkalosis. Clin. Sci. 29:159-170.

2. de Mello Aires, M., and G. Malnic. 1972. Micropuncture study of acidification during hypochloremic alkalosis in the rat. Pfluegers Arch. Eur. J. Physiol. 331:13-24.

3. de Mello Aires, M., and G. Malnic. 1972. Renal handling of sodium and potassium during hypochloremic alkalosis in the rat. Pfluegers Arch. Eur. J. Physiol. 331:215-225.

4. Cogan, M. G., and F. L. Lui. 1983. Metabolic alkalosis in the rat. Evidence that reduced glomerular filtration rather than enhanced tubular bicarbonate reabsorption is responsible for maintaining the alkalotic state. J. Clin. Invest. 71:1141-1160.

5. Maddox, D. A., and F. J. Gennari. 1983. Proximal tubular bicarbonate reabsorption and $\mathrm{PCO}_{2}$ in chronic metabolic alkalosis in the rat. J. Clin. Invest. 72:1385-1395.

6. Maddox, D. A., and F. J. Gennari. 1986. Load dependence of proximal tubular bicarbonate reabsorption in chronic metabolic alkalosis in the rat. J. Clin. Invest. 77:709-716.

7. Schnermann, J., F. S. Wright, J. M. Davis, W. V. Stakelberg, and G. Gill. 1970. Regulation of superficial nephron filtration rate by tubuloglomerular feedback. Pfluegers Arch. Eur. J. Physiol. 318:147175.

8. Wesson, D. E. 1987. Glomerular filtration effects of acute volume expansion. Importance of chloride. Kidney Int. 32:238-245.

9. Seldin, D. W., and F. C. Rector, Jr. 1972. The generation and maintenance of metabolic alkalosis. Kidney Int. 1:306-321.

10. Kurtzman, N. A. 1970. Regulation of renal bicarbonate reabsorption by extracellular volume. J. Clin. Invest. 49:586-595.

11. Kaufman, A. M., and T. Kahn. 1988. Complementary role of citrate and bicarbonate excretion in acid-base balance in the rat. Am.J. Physiol. 255 (Renal Fluid Electrolyte Physiol. 24):F182-F187.

12. Wesson, D. E. 1987. Hypocalcemia-associated modulation of renal response to acute volume expansion in rats. Am. J. Physiol. 253 (Renal Fluid Electrolyte Physiol. 22):F726-F733.

13. Liu, F. Y., and M. G. Cogan. 1986. Axial heterogeneity of bicarbonate, chloride, and water transport in the rat proximal convoluted tubule. J. Clin. Invest. 78:1547-1557.

14. Kruhoffer, P. 1946. Inulin as an indicator for the extracellular space. Acta Physiol. Scand. 11:16-36. 
15. Vurek, G. G., D. G. Warnock, and R. Corsey. 1975. Measurement of picomole amounts of carbon dioxide by microcalorimetry. Anal. Chem. 47:765-767.

16. Cogan, M. G., D. A. Maddox, M. S. Lucci, and F. C. Rector, Jr. 1979. Control of proximal bicarbonate reabsorption in normal and acidotic rats. J. Clin. Invest. 64:1168-1180.

17. Haberle, D. A., T. T. Shiigai, G. Maier, H. Schiffl, and J. M. Davis. 1981. Dependency of proximal tubular fluid transport on the load of glomerular filtrate. Kidney Int. 20:18-28.

18. Peterson, O. W., L. C. Gushwa, and R. C. Blantz. 1986. An analysis of glomerular tubular balance in the rat proximal tubule. Pfluegers Arch. Eur. J. Physiol. 407:221-227.

19. Alpern, R. J., M. G. Cogan, and F. C. Rector, Jr. 1983. Flow dependence of proximal tubular bicarbonate reabsorption. Am. J. Physiol. 245 (Renal Fluid Electrolyte Physiol. 14):F478-F484.

20. Capasso, G., R. Kinne, G. Malinc, and G. Giebisch. 1986. Renal bicarbonate reabsorption in the rat. I. Effects of hypokalemia and carbonic anydrase. J. Clin. Invest. 78:1558-1567.

21. Liu, F. L., and M. G. Cogan. 1987. Kinetics of bicarbonate transport in the early proximal convoluted tubule. Am. J. Physiol. 253 (Renal Fluid Electrolyte Physiol. 22):F912-F916.
22. Matsamura, Y., B. Cohen, W. B. Guggino, and G. Giebisch. 1984. Regulation of the basolateral potassium conductance of the Necturus proximal tubule. J. Membr. Biol. 79:153-161.

23. Cemerikic, D., C. S. Wilcox, and G. Giebisch. 1982. Intracellular potential and $\mathrm{K}^{+}$activity in rat kidney proximal tubular cells in acidosis and $\mathrm{K}^{+}$depletion. J. Membr. Biol. 75:159-165.

24. Chan, Y. L., B. Biagi, and G. Giebisch. 1982. Control mechanisms of bicarbonate transport across the rat proximal convoluted tubule. Am. J. Physiol. 242:F532-F543.

25. Alpern, R. J., M. G. Cogan, and F. C. Rector, Jr. 1983. Effects of extracellular fluid volume and plasma bicarbonate concentration on proximal acidification in the rat. J. Clin. Invest. 71:736-746.

26. Galla, J. H., D. N. Bonduris, and R. G. Luke. 1983. Correction of acute chloride-deplete alkalosis in the rat without volume expansion. Am. J. Physiol. 244 (Renal Fluid Electrolyte Physiol. 13):F217F221.

27. Wall, B. M., G. V. Byrum, J. H. Galla, and R. G. Luke. 1987. Importance of chloride for the correction of chronic metabolic alkalosis in the rat. Am. J. Physiol. 253 (Renal Fluid and Electrolyte Physiol. 22):F1031-F1039. 